\title{
PREFACE
}

\section{Phenotyping for early predictors of obesity and the metabolic syndrome}

\author{
AG Dulloo and J-P Montani \\ Department of Medicine/Physiology, University of Fribourg, Fribourg, Switzerland
}

International Journal of Obesity (2010) 34, S1-S3; doi:10.1038/ijo.2010.233

The continuing surge in the global prevalence of obesity, which is the driving force behind the projected pandemic of type 2 diabetes and its cardiovascular complications by $2030,{ }^{1,2}$ is certainly due to socioeconomic, cultural and other environmental changes that promote overeating and discourage physical activity. However, there are many individuals exposed to these same obesogenic and diabetogenic environments who, apparently without conscious effort, do not become obese, and still others who are obese but metabolically healthy in that they do not show risks for the metabolic syndrome. One can of course ignore the importance of genes in these variabilities, and rather prefer to invoke the importance of family, social and cultural habits (including learnt lifestyle behaviors) as having a protective role against their 'obesogenic' or 'diabetogenic' environments. But equally valid are the arguments that lifestyle effects and behaviors may also be inherited, thereby propelling us into the complex area of gene-environment interactions. These interactions between nature and nurture are expressed through a multitude of observable characteristics or traits (that is, phenotypic outcomes), some of which may have diagnostic (identification) and prognostic (predictive) values pertaining to obesity and its co-morbidities. The enormous challenges faced by the researchers in the field of human phenotyping today can be encapsulated by three questions: What to phenotype? How to phenotype? When to phenotype? These are the fundamental questions that are addressed in this $I J O$ supplement reporting the proceedings of the Fifth Fribourg Obesity Research Conference (FORC-2009) that was held in Fribourg, Switzerland, on the theme of 'Phenotyping for early predictors of obesity and the metabolic syndrome'. This 1-day conference was attended by about 100 researchers from both academia and industry, and was divided into three sessions of three lectures each (hence a total of 9 articles in this supplement), as

Correspondence: $\mathrm{Dr}$ AG Dulloo, Department of Medicine/Physiology, University of Fribourg, Fribourg, Switzerland.

E-mail: abdul.dulloo@unifr.ch well as poster sessions, the Abstracts of which are also published at the end of this supplement.

\section{Session I: Body composition, metabolic health and ethnicity}

Obesity is defined as an excess of adipose tissue mass, but current classifications of obesity and its co-morbidities are based on body mass index (BMI), waist circumference and other anthropometric measures, which, although useful for population studies, have considerable limitations in the assessment of body composition and lack sensitivity for assessing disease risks, particularly in people who have normal or mildly elevated body weight. After a critical examination of these limitations within the constraints of the relationship between BMI and \% body fat, Dulloo et al. review recent advances in concepts about health risks related to body composition phenotypes centered on the partitioning of BMI into a fat mass index and a fat-free mass index. The partitioning of fat-free mass into organ mass and muscle mass, the anatomical partitioning of fat mass into hazardous fat and protective fat, and the interplay between adipose tissue expandability and ectopic fat deposition within or around organs/tissues that constitute the lean body mass are also considered. These concepts about body composition phenotypes and health risks are reviewed in the light of ethnic variability in metabolic susceptibility to obesity and the metabolic syndrome. This session also addresses another obesity classification issue, namely, pertaining to how to better define the subset of obese individuals who apparently do not present overt cardiometabolic risk factors generally associated with increased fatness. By raising 'open questions about metabolically normal obesity', Pataky et al. underscore the need to think and reflect about establishing a universal definition for metabolic normality in obese individuals, and the need to search for determinants that would distinguish metabolically normal obesity from obesity with features of the 
metabolic syndrome. There are also many open questions about why the aboriginal populations in many parts of the world carry a much higher risk for obesity and type 2 diabetes than their Caucasian counterparts. Similar to the Pima Indians living in Arizona, the First Nations communities of Northern Ontario (primarily Ojibwe, Cree and Oji-Cree nations) have been experiencing an alarming rise in obesity and type 2 diabetes, with symptoms occurring early during adulthood. In the review of research conducted on these diabetes-prone communities, often under the inhospitable conditions of 'Le Grand Nord', Haman et al. explore the biological predispositions and potential environmental factors (including food chain contaminants and environmental pollutants) that contribute to the development of the high incidence of obesity and obesity-related diseases in these communities.

\section{Session II: Obesity and disease risks in mothers and children}

The surge in childhood overweight worldwide poses a major additional burden on public health insofar as 'adult' diseases manifest themselves already during childhood, thereby exposing children to the potentially damaging metabolic and cardiovascular insults during critical periods of growth and development. After examining the prevalence of metabolic and cardiovascular diseases in obese children, L'Allemand addresses the issue of whether BMI or other clinical parameters are valid tools to predict co-morbidities in children, and furthermore argues for the necessity of screening for cardiovascular risk factors even in children of normal BMI but with elevated waist circumference.

The need for early detection of psychological factors contributing to the onset and maintenance of obesity is also emphasized by Puder and Munsch. They underscore the need for more research into the interactions between behavior, cognition and emotional regulation within the family structure, and take into account the inter-relatedness between psychological problems and childhood obesity within treatment procedures. Still, in the context of the family, the prevalence of overweight and obese pregnant women is growing with the overall obesity epidemic. Although there is little known in humans about the influences of overweight and obesity during pregnancy and lactation on the health of both the infant and the mother, data in animal models of maternal obesity are unequivocal: maternal obesity during pregnancy and lactation impacts strongly on developmental programming of the offspring, rendering them more susceptible to later insulin-related disorders. Furthermore, as pregnancy weight gain is also a risk factor for later obesity in the mother, there is an increasing need for promoting counseling before, during and after pregnancy on the role of diet and physical activity in reproductive health. In their review on this topic, Melzer and Schutz analyze the factors that may predict development of obesity in later life, including gestational weight gain, pre-pregnancy nutritional status, age, parity and race. They also discuss the changes in lifestyle factors, such as eating habits, enrolment in physical activity, smoking and duration of lactation, as potential contributory factors to the development of obesity.

\section{Session III: High-tech phenotyping}

The availability of genetically altered mice has brought a considerable number of new ways to explore basic physiological functions, but the small size of the mouse and the environmental conditions to which the mice are exposed have major repercussions on the phenotyping outcome. As Overton points out in his review on the topic of phenotyping small animals, it is standard practice in preclinical biomedical research to house mammalian model organisms at an ambient temperature at which humans are comfortable, but which is substantially below the thermoneutral zone for small mammals, that is, under conditions when they are chronically challenged by mild cold stress. He reviews the physiology associated with activation of non-shivering thermogenesis and analyzes how this activation can influence common strategies used to phenotype genetically engineered mice (and in the near-future rats) as model organisms to understand the metabolic syndrome in humans. He also counters the argument that 'putting clothes on mice to bring them to thermoneutrality would be neither practical or prudent.' by proposing that it may be both practical and prudent to change the thermostat in the room housing these mice. It is indeed indisputable that the currently accepted strategy of housing mice in cool rooms fundamentally alters nearly all physiological systems associated with the metabolic syndrome. Paradoxically, it was the unexpected outcome of exposure of humans to mild cold during routine positron emission tomography/computed tomography scanning in nuclear medicine hospital units that has recently led to the resolution of one of the most controversial extrapolations of a mouse phenotype to humans: the presence, in adult men and women, of functional brown adipose tissue. As its primary physiological role is to produce heat, the (re)discovery that brown adipose tissue can be reactivated within $1-2 \mathrm{~h}$ of cold exposure in adult humans has provided a 'hot target' for pharmaceutical (and nutraceutical) companies active in the development of thermogenic compounds for antiobesity and antidiabetic therapy. Richard et al. review the recent progress made in the biology of the brown fat cell (which is the only cell type in the body that possesses a physiologically relevant uncoupling protein for thermogenesis) and focus on the determinants of the brown adipocyte development and activity from mice to humans. Whether the application of nuclear medicine technology to monitor brown adipose tissue activity in humans will eventually be replaced by techniques that expose the subjects to less (or no) radiation remains to be seen. However, the use of modern imaging techniques such as computer tomography and magnetic resonance imaging, which have already revolutionized deep phenotyping of body 
composition and fat distribution, promises to provide more sensitive diagnostic tools for the early detection of metabolic and cardiovascular dysfunction. As Montet et al. emphasize in their review on this rapidly advancing field, the techniques of magnetic resonance imaging and magnetic resonance spectroscopy provide a non-invasive, well-suited approach to evaluate cardiovascular pathophysiology and cardiac function at the whole organ level (by cine-magnetic resonance imaging), the regional level (through tag, perfusion and viability imaging), the metabolic level (with magnetic resonance spectroscopy) and at the cellular and even molecular level (with molecular imaging). These techniques are promising for guiding diagnosis and therapy, and will allow early detection of cardiovascular diseases and better risk assessments.

\section{Conclusion}

This supplement contains a set of nine review articles that integrate, from a perspective of system physiology and nutrition, some of the recent advances and emerging concepts about phenotyping for early predictors of obesity and metabolic syndrome in view of developing effective strategies for prevention and treatment monitoring. The challenges are enormous, but the call for 'The Human Phenome Project' ${ }^{3}$ signifies a post-genomic era ready to tackle complexity rather than simplification in the relationship between genotype and phenotype.

\section{Conflict of interest}

The authors declare no conflict of interest.

\section{References}

1 Wild S, Roglic G, Green A, Sicree R, King H. Global prevalence of diabetes: estimates for the year 2000 and projections for 2030. Diabetes Care 2004; 27: 1047-1053.

2 Kelly T, Yang W, Chen CS, Reynolds K, He J. Global burden of obesity in 2005 and projections to 2030 . Int J Obes 2008; 32: 1431-1437.

3 Freimer N, Sabatti C. The human phenome project. Nat Genet 2003; 34: 15-21. 